\title{
Early Institutional Experience of Management of Ruptured Intracranial Aneurysms: Our Team Approach
}

\author{
Salek MAA ${ }^{1}$, Shariar $\mathbf{R}^{1}$, Faisal $\mathbf{M H}^{1}$, Manik $\mathrm{MAH}^{1}$, Choudhury $A \mathrm{UM}^{1}{ }^{1}$, Chowdhury $\mathrm{RU}^{1}$, \\ Islam MA ${ }^{1}$, Ahmed $M^{2}$, Alam $Z^{3}$, Islam $S^{4}$
}

\author{
Conflict of interest: There is no conflict \\ of interest relevant to this paper to \\ disclose.
}

Funding Agency: Was not funded by any institute or any group.

Contribution of Authors: Salek MAA was Principal investigator, Shariar $\mathrm{R}$ help for protocol preparation, Faisal $\mathrm{MH}$, Manik MAH, Choudhury AUM, Chowdhury RU, Islam MA, Ahmed M, Alam Z help for data collection and Islam $S$ help for editorial formatting.

Copyright: @2019 Bang. JNS published by BSNS. This article is published under the creative commons CC-BY-NC license.

This license permits use distribution (https://creativecommons.orgf/ licences/by-nc/4-0/) reproduction in any medium, provided the original work is properly cited, and is not used for commercial purposes.

Received: 27 February, 2019 Accepted: 11 March, 2019

\begin{abstract}
Introduction: Intracranial aneurysms are relatively common, with a prevalence of approximately 4\%. Rupture of an intracranial aneurysm (IA) causing subarachnoid haemorrhage (SAH) is a devastating event that is still associated with a $50 \%$ case fatality rate, despite major improvements in surgical techniques, diagnosis and interventional treatment. While patient subgroups may clearly qualify for either surgical or endovascular treatment some patients could benefit from multimodal therapy. In this article we will show our team approach experience of management of ruptured intracranial aneurysms.
\end{abstract}

Methods: Cross-sectional descriptive study of 52 patients of ruptured intracranial aneurysm who got admitted into Combined Military Hospital, Dhaka in the period from Jan 2016 to Dec 2018. Patients were evaluated according to their demographic data, analysis of risk factors, radiological location of aneurysms, World Federation of Neurological Surgeon (WFNS) scale, procedural options for aneurysm treatment, post procedural complications. We excluded unruptured cases and those patients who were treated conservatively. General outcome was assessed through the Glasgow out-come (GOS) scale. Data analysis was done by using computer software SPSS version 25.0.

Patients studied were mainly middle-aged with mean age $44 \pm 5.2$ years and predominantly female. Most of the patients had previous history of hypertension 45 (86.53\%). WFNS scale 2 was observed in 34 (65.38\%). Anterior communicating (Acom) artery aneurysm was more frequent which is found in 16 (30.77\%) cases. 37 (71.15\%) patients underwent microsurgical clipping whereas 13 (25\%) patients underwent endovascular intervention and 2 (3.85\%) patients needed combined approach. Complications were more in microsurgical clipping group 38 (73.08\%) in comparison to endovascular group 13 (25\%). GOS scale 5 was observed in $30(57.70 \%)$ cases.

Conclusion: Our experience of management of ruptured intracranial aneurysms together with their outcome have been reflected in this small study. For better outcome of ruptured intracranial aneurysms decision can be made on an individual case by case basis. Hybrid treatment option of staged endovascular and open microsurgical proceduresare sometimes needed as a combined approach.

Key words: intracranial aneurysm, subarachnoid haemorrhage, neurointervention, rebleeding, vasospasm, hybrid treatment option.

Bang. J Neurosurgery 2019; 9(1): 39-43

\section{Introduction}

Intracranial aneurysms (IAs) are vascular lesions that can rupture and lead to subarachnoid haemorrhage $(\mathrm{SAH})$ and this poses a challenge for decision making and management. Several factors have to be taken into consideration to decide the optimal treatment approach, including the natural history of aneurysms and risk factors for aneurysm formation, growth, and

1. Md Al Amin Salek, Rajib Shariar, Md Hasnain Faisal, Md Abdul Hye Manik, Ahmed-Ul-Mursalin Choudhury, Rukun Uddin Chowdhury, Md Aminul Islam, Department of Neurosurgery, Combined Military Hospital Dhaka, Bangladesh

2. Masud Ahmed, Department of Anaesthesiology, Combined Military Hospital Dhaka, Bangladesh

3. Zoherul Alam, Department of Radiology, Combined Military Hospital Dhaka, Bangladesh

4. Shafiqul Islam, Department of Neurosurgery, Dhaka Medical College and Hospital

Address of Correspondence: Lt Col Md Al Amin Salek, MBBS, MCPS, FCPS, MRCS, Department of Neurosurgery, Combined Military Hospital Dhaka, Email: salek1972@yahoo.com 
rupture. Intracranial aneurysms are diagnosed and monitored with imaging including intra-arterial digital subtraction angiography, computed tomography angiography, magnetic resonance angiography, and recently transcranial doppler ultrasonograpy has been proposed as a potential modality ${ }^{1}$. Treatment options include observation, endovascular coiling, surgical clipping and combination approach. In this paper the subgroups of ruptured intracranial aneurysm patients are studied in relation of epidemio-clinical and treatment outcome.

\section{Method:}

We carried out cross-sectional observational study of 52 consecutive patients with ruptured intracranial aneurysm admitted in the Department of Neurosurgery, Combined Military Hospital Dhaka in the period between January 2014 and December 2018. All those patients who were admitted and received either endovascular and or microsurgical clipping of one or more aneurysms were eligible for the study. Patients with non-ruptured aneurysm and those who received conservative treatment were excluded from the study. History for risk factors such as age, gender, history of hypertension or smoking habit were obtained from the patient or close relatives. The clinical presenting condition was defined as per the WFNS scale (Fig-1). Anatomical location by radiological data were obtained through $\mathrm{CT}$ angiogram and digital subtraction angiography (DSA). Both the SAH and procedure related complications were studied. General out come and degree of disability were defined according to the GOS and rated in three to six-month outpatient follow-up except in cases of death. Algorithm for evaluation of ruptured intracranial aneurysm (Fig-2) and decision for multimodal treatment options(Fig-3) were used.

\begin{tabular}{lcc}
\hline Grade & $\begin{array}{c}\text { Glasgow coma } \\
\text { Scale score }\end{array}$ & Motor deficit \\
\hline I & 15 & Absent \\
II & $14-13$ & Absent \\
III & $14-13$ & Present \\
IV & $12-7$ & Present or absent \\
V & $6-3$ & Present or absent \\
\hline
\end{tabular}

WFNS = World Federation of Neurological Surgeons'

Fig.-1: Clinical grade of subarachnoid haemorrhage

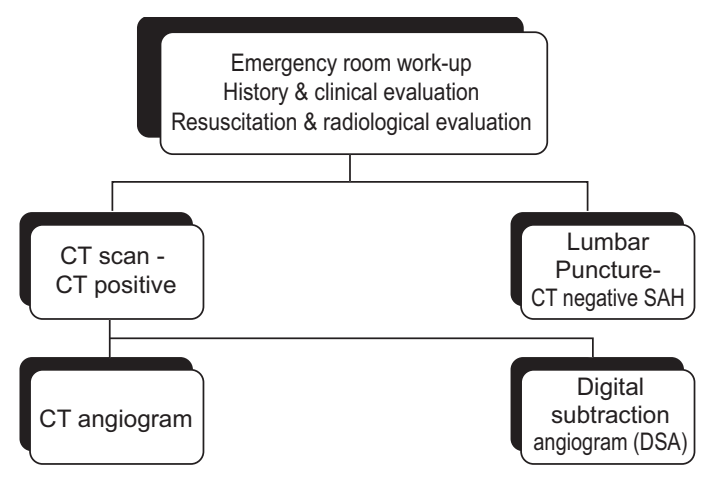

Fig.-2: Algorithmic evaluation of ruptured intracranial aneurysm

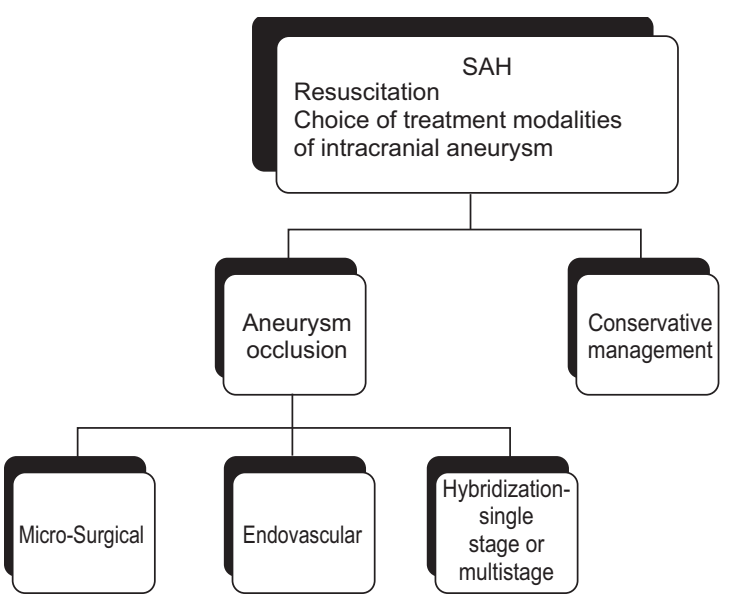

Fig.-3: Algorithmic decision for multimodal treatment options of ruptured intracranial aneurysm

\section{Results:}

Distribution of sex- In our study we observed female preponderance which is shown in Fig-4.

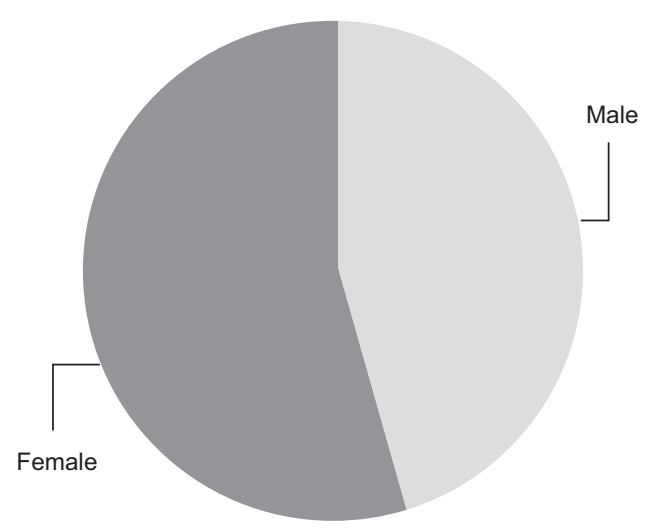

Fig- 4: Distribution of sex

Distribution of age- Study group age range was 10-80 years with most frequent in 41-50 years group 24 $(46.15 \%)$ as shown in Table-I. 
Table-I

Distribution of age

\begin{tabular}{lcc}
\hline Age range (years) & Frequency & Percentage \\
\hline $10-20$ & 1 & 1.92 \\
$21-30$ & 3 & 5.77 \\
$31-40$ & 6 & 11.54 \\
$41-50$ & 24 & 46.15 \\
$51-60$ & 11 & 21.15 \\
$60-70$ & 5 & 9.62 \\
$70+$ & 2 & 3.85 \\
\hline
\end{tabular}

Risk factors - A majority of patients had a previous history of essential hypertension $45(86.53 \%)$ and 8 $(15.38 \%)$ patents had history of cigarette smoking (Table-II).

Table-II

Distribution of risk factors

\begin{tabular}{lcc}
\hline Risk factors & Frequency & Percentage \\
\hline Hypertension & 45 & 86.53 \\
Cigarette smoking & 8 & 15.38 \\
Alcohol consumption & 1 & 1.92 \\
Straining & 2 & 3.85 \\
\hline
\end{tabular}

Clinical condition- WFNS scale 2 was the most frequent $34(65.38 \%)$ (Table-III).

Table-III

Clinical condition according to the WFNS scale

\begin{tabular}{lcc}
\hline WFNS grade & Frequency & Percentage \\
\hline 1 & 33 & 63.46 \\
2 & 34 & 65.38 \\
3 & 25 & 48.08 \\
4 & 6 & 8.82 \\
5 & 2 & 3.85 \\
\hline
\end{tabular}

Radiological location- Anteriorly located aneurysms were most prevalent (67.30\%)than posteriorly located $(32.70 \%)$. Anatomical site of the intracranial aneurysm was observed according to table-IV.

Aneurysm treatment options- Among endovascular procedures $12(23.08 \%)$ patient underwent coil embolization while 1 patient could be treated by glue embolization. In microsurgical group clipping was done in $34(65.38 \%)$ patients, wrapping in $2(3.85 \%)$ patients (unsuitable for clipping) where as one could be treated by ligation and excision of a ten years old boy. 2 $(3.85 \%)$ patents were treated as staged hybridization for incomplete occlusion by a single technique (TableV).

Complications - Both SAH related and procedure related complications are summarized in table-VI.

Among microsurgical complications, clinical or radiological vasospasm was observed in $5(9.62 \%)$ cases, rebleeding in $2(3.84 \%)$ cases and metabolic and infective events in $8(15.38 \%)$ cases. There was convulsion in $3(5.77 \%)$ cases, hydrocephalus in 5 $(9.62 \%)$ cases, neurological deterioration in 6 $(11.54 \%)$ cases. Death occurred in $5(9.62 \%)$ cases.

In endovascular group there was puncture site haematoma in one patient, post-procedural metabolic and infection derangement observed in $(7.69 \%)$ patients. Coil prolapses occurred in $2(3.84 \%)$ patients in which one patient need microsurgical clipping for incomplete occlusion. Death occurred in 2 (3.84\%)patients.

General outcome - Within a three to six-month period of outpatient follow-upGlasgow out-come scale (GOS) 5 is the most frequent in $30(57.70 \%)$ (Table-VII).

Table-IV

Anatomical site of the intracerebral aneurysms.

\begin{tabular}{lcc}
\hline Site of aneurysm & Frequency & Percentage \\
\hline Middle cerebral artery (MCA) & 10 & 19.23 \\
Posterior communicating artery (P-Com) & 13 & 25 \\
Anterior communicating artery (A-Com) & 16 & 30.77 \\
Internal carotid artery (ICA) & 7 & 13.46 \\
Basilar artery (BA) & 3 & 5.77 \\
Posterior inferior cerebellar artery (PICA) & 1 & 1.92 \\
Distal Anterior Cerebral artery (DACA) & 2 & 3.85 \\
\hline
\end{tabular}


Table-V

Treatment options of the intracerebral aneurysms.

\begin{tabular}{lcl}
\hline Therapeutic options & Frequency $(\%)$ & Remarks \\
\hline Endovascular & $13(25)$ & \\
Coiling & $12(23.08)$ & \\
Glue Embolization & $1(1.92)$ & DACA \\
Microsurgical & $37(71.15)$ & \\
Clipping & $34(65.38)$ & \\
Wrapping & $2(3.85)$ & Ruptured friable neck of A-Com, ICA aneurysm \\
& & M3/M4 aneurysm in young boy \\
Ligation and excision & $1(1.92)$ & Staged hybridization \\
Hybrid & $2(3.85)$ &
\end{tabular}

Table-VI

Complications

\begin{tabular}{lcl}
\hline Complications & Frequency (\%) & Remarks \\
\hline Microsurgical & & *Among hydrocephalus three \\
Clinical and radiological vasospasm & $5(9.62)$ & needed external CSF diversion, \\
Rebleeding & $2(3.84)$ & and two remained shunt \\
Metabolic and infective & $8(15.38)$ & dependent. \\
Convulsion & $3(5.77)$ & \\
Hydrocephalus & $5(9.62)$ & *Out of five deaths one occurred \\
Neurological deterioration & $6(11.54)$ & during surgery \\
Incomplete occlusion & $1(1.92)$ & (intra-op rupture), one due to \\
Death & $5(9.62)$ & vasospasm and remaining three \\
& & due to metabolic and infective events. \\
Endovascular & & * Out of two coil prolapses one \\
Puncture site haematoma & $1(1.92)$ & needed adjunct microsurgery \\
Coil prolapses & $2(3.84)$ & \\
Incomplete occlusion & $1(1.92)$ & * Two deaths occurred post \\
Metabolic and infective & $4(7.69)$ & procedure cardiac arrest \\
Death & $2(3.84)$ & \\
\hline
\end{tabular}

*Multiple findings

Table-VII

GOS outcome

\begin{tabular}{lcc}
\hline GOS & Frequency & Percentage \\
\hline 1 & 7 & 13.46 \\
2 & 2 & 3.85 \\
3 & 6 & 11.54 \\
4 & 7 & 13.46 \\
5 & 30 & 57.70 \\
\hline
\end{tabular}

\section{Discussion:}

Rupture of an intracranial aneurysm is one of the most common neurosurgical emergencies, with an incidence of approximately 6-10 in 100000 persons per year ${ }^{2}$.
Despite major advances in the understanding and treatment of this condition over the last 30 years, the prognosis remains poor, with an overall mortality of $30 \%$ or more, and less than $50 \%$ of patients making a full functional recovery ${ }^{3}$. Although clipping of a ruptured intracranial aneurysm has long been regarded as the gold standard in the treatment of this condition, a new mode of treatment, namely endovascular coiling of the aneurysm, has been increasingly employed since the second half of the 1990s.

Arterial hypertension coexists in high percentage of patientsand it has been proposed as an etiological factor, even though the exact relationship is not completely defined. Cigarette smoking is the single 
acquired factor that has been proved to confer risk for aneurysmal subarachnoid hemorrhage ${ }^{4}$. In our study we have found association of hypertension in $86.53 \%$ of cases.

In radiological location of ruptured intracranial aneurysms (RIAs) we found A-Com (30.77\%), P-Com (25\%) and MCA (19.23\%). MiikkaKorja et al ${ }^{5}$ found the 4 most common locations of RIAs were the middle cerebral $(32 \%)$, anterior communicating $(32 \%)$, posterior communicating (14\%), and pericallosal arteries (5\%).

As per International Subarachnoid Aneurysm Trial (ISAT) endovascular coiling gives rise to immediate results that are superior to surgery for the majority of aneurysms ${ }^{6}$. Quite apart from this, there are clear advantages to coiling, such as a shorter hospital stay, the avoidance of open surgical manipulation of the brain, and the absence of postoperative epilepsy. But in our study, we applied microsurgical clipping (71.15\%) more than endovascular therapy $(25 \%)$ due to constraint of resources and lack of experience.

We found a 3 years mortality of $9.62 \%$ in microsurgical clipping and $3.84 \%$ in endovascular coiling. The reported results of aneurysm surgery vary widely, with an operative mortality from $3 \%$ to $36 \%{ }^{7}$.

In our present series, morbid complications occurred in $73.06 \%$ of patients. Although the great majority of patients recovered from these complications, it is likely that coiling will eliminate most such complications ${ }^{8}$.

Post treatment GOS 4 and 5 together comprise $71.18 \%$. These results are comparable with other surgical series with a similar case ${ }^{9}$. However, these management results do not represent the whole picture, as many additional patients would have died before transfer to the neurosurgical unit, and the delays in transfer would have had the effect of lowering the management mortality.

The main causes of poor outcome in patients with $\mathrm{SAH}$ are effects of bleeding ${ }^{10}$.Progress in surgical techniques,early surgical approach and clinical advances have consistently decreased morbidity and morbodity and mortality ${ }^{11}$.Other factors such as delayed cerebral ischemia, hydrocephalus and rebleeding have been correlated to degree of disability.

\section{Conclusion:}

Decisions regarding how best to treat an aneurysm patient are always specific to that particular patient and need to be made on an individual case by case basis. Endovascular occlusion of the ruptured intracranial aneurysms can be considered prior to microsurgical clipping owing to its less procedure related complications, but sometimescombined hybrid procedures are needed for selective cases of ruptured intracranial aneurysms.

\section{References:}

1. Keedy A. An overview of intracranial aneurysms. Mcgill J Med. 2006;9(2):141-146.

2. Investigators TISoUIA. Unruptured intracranial aneurysms-risk of rupture and risks of surgical intervention. International Study of Unruptured Intracranial Aneurysms Investigators. N Engl J Med 1998; 339:172533.

3. Linn FH, Rinkel GJ, Algra A, et al. Incidence of subarachnoid hemorrhage: Role of region, year, and rate of computed tomography: a meta-analysis. Stroke 1996; 276:25-9.

4. Bonita R. Cigarette smoking, hypertension and the risk of subarachnoid hemorrhage: a population-based case contral study.Stroke 1986:17:831 835.

5. MiikkaKorja, RikuKivisaari, Behnam RezaiJahromi and Hanna Lehto. Size and location of ruptured intracranial aneurysms: consecutive series of 1993 hospital-admitted patients. Journal of Neurosurgery 2017; 127:709-964.

6. International Subarachnoid Aneurysm Trial (ISAT) collaborative group. International Subarachnoid Aneurysm Trial (ISAT) of neurosurgical clipping versus endovascular coiling in 2143 patients with ruptured intracranial aneurysms: A randomized trial. Lancet2002; 360:126774.

7. Auer LM. Unfavorable outcome following early surgical repair of ruptured cerebral aneurysms-a critical review of 238 patients. Surg Neurol1991; 35:152-8.

8. Leber KA, Klein GE, Trummer M, et al. Intracranial aneurysms: a review of endovascular and surgical treatment in 248 patients. Minim Invasive Neurosurg 1998; $41: 81-5$

9. Hackett ML. Anderson CS. Health outcomes 1 year after subarachnoid hemorrhage: An international populationbased study. The Australian cooperative Research on subarachnoid Hemorrhage study Group.Neurology 2000:55:658 662 .

10. Roos YB. de HaanRj. Beenen LF. Groen Rj.Albrecht KW. Vermeulen M. complications and outcome in patients with aneurysmal subarachnoid haemorrhage: A prospective hospital-based cohort study in the Netherlands. J Neurol Neurosurg psychiatry 2000:68:337 341 .

11. Hutter BO. KrackP. Buchinger and Ermahr.Mayfrank L. Rohde V. SpetzgerU. Hilsbach JM.Functional outcome after aneurysmal subarachnoid hemorrhage.Acta neurochir(Wien)1999:72(suppl):157 174. 